\title{
Reordenación de la mortalidad en el noroeste de México, según el índice de años de vida productivos perdidos
}

Ramón Alberto Rascón-Pacheco, M.C., M. en C., ${ }^{(1)}$ Blanca Margarita Rivera-Icedo, Lic. en Psic., (1)

Manuel Alberto Santillana-Macedo, M.C., M. en C., (1) Miguel Bernardo Romero-Téllez, M.C. ${ }^{(2)}$

\section{Rascón-Pacheco RA, Rivera-Icedo BM, Santillana-Macedo MA, Romero-Téllez MB. Reordenación de la mortalidad en el noroeste de México, según el índice de años de vida productivos perdidos. Salud Publica Mex 1999;41:376-380.}

\section{Resumen}

Objetivo. D eterminar el orden de causas de muerte en las entidades federativas de la región noroeste de México, utilizando el índice de años de vida productivos perdidos (AVPPipc). Material y métodos Se utilizó la base de datos elaborada por el Instituto $\mathrm{N}$ acional de Estadística, G eografía e Informática, so bre mortalidad en Baja California, Baja C alifornia Sur, Sinaloa y Sonora para 1995. Se siguieron los pasos de Bustamante y colaboradores para ponderar las defunciones entre la inversión, la producción y el consumo potencial de cada individuo, de acuerdo con la etapa de productividad en que los individuos fallecen. Resultados Al utilizar el indicador AVPPipc se reordenaron las causas de muerte. En las cuatro entidades federativas el grupo de edad con mayor pérdida potencial es el de 5 a 14 años, sobre todo en Baja California y Sonora. De acuerdo con la IX Clasificación Internacional de Enfermedades, el grupo de causas " $C$ iertas afecciones originadas en el periodo perinatal" ocupa el primer lugar en tres de las entidades federativas, y el de "H omicidio s y lesiones infligidas intencio nalmente por otra persona" es el primero en Sinaloa. Conclusiones. Este trabajo resalta el hecho de que al utilizar un indicador de años potencialmente perdidos con una ponderación económica, se puede hacer una discriminación entre las entidades federativas, dentro de una región aparentemente homogénea en la mortalidad.

Palabras clave: índice años de vida productivos perdidos; valor económico de la vida; mortalidad; planificación en salud; regionalización; México

\author{
Rascón-Pacheco RA, Rivera-Icedo BM, \\ Santillana-Macedo MA, Romero-Téllez MB. \\ Mortality ranking in the Northwest \\ of Mexico according to the years \\ of potential life lost index.
}

Salud Publica Mex 1999;41:376-380.

\begin{abstract}
A bstract
Objective.To determine the order of death causes in the $\mathrm{N}$ or thwestern states of Mexico using the years of po tential life lost (YPLL) index. Material and methods. The 1995 IN EG I mortality data base for the states of Baja California, Baja California Sur, Sinaloa and Sonora was used to estimate the YPLL index. The method by Bustamante et al. was followed to calculate the cause of death, with ponderation of the potential investment, production and consumption of each individual, according to the age of death. Results. A ranking of cause of death resulted from the application of the YPLL index.At present, in the four states, the group with the greatest potential loss is that between 5 and 14 years of age, and the rate is higher in Baja C alifornia than in Sonora.The group of "Perinatal problems as a cause of death" ranked first in three states and in the fourth, Sinaloa, the group of "Homicide and lesions caused intentionally by another person" ranked first. ConclusionsThis work highlights the importance of the YLPP with economic loss ponderation index to discriminate death causes among states in a region with apparently homogeneous mortality.
\end{abstract}

Key words: years of potential life lost; economic value of life; mortality; health planning; regional health planning; Mexico

(1) Unidad de Investigación Epidemiológica y en Servicios de Salud, Instituto Mexicano del Seguro Social (IMSS), Hermosillo, Sonora, México.

(2) Delegación del IMSS en Sonora, México.

Fecha de recibido: 18 de marzo de 1999 • Fecha de aprobado: 14 de julio de 1999

Solicitud de sobretiros: Dr. Ramón Alberto Rascón Pacheco. Unidad de Investigación Epidemiológica y en Servicios de Salud. Instituto Mexicano del Seguro Social. Juárez y Seguro Social s/n, colonia Modelo, 83150 Hermosillo, Sonora, México.

Correo electrónico: arascon@ son1.telmexnet.mx 
$\mathrm{T}$ anto para los académicos dedicados a la investigación social, como para los planificadores públicos y privados, es importante conocer con mayor especificidad el comportamiento actual de la mortalidad y sus consecuencias. ${ }^{1}$ La mortalidad, la salud y la población son temas que tienden a discutirse asociados, debido a las numerosas relaciones que existen entre ellos. Esto permite conocer algunas causas de mortalidad desde diferentes perspectivas y, adicionalmente, proponer estrategias dirigidas a atenderlas.

Cuando los recursos disponibles para la atención social son limitados, el proceso de planeación debe comenzar por el reconocimiento de las necesidades poblacionales. Posteriormente, se deben escoger las alternativas que permitan cumplir con los objetivos fijados, minimizando el consumo de recursos y tiempo; ${ }^{2}$ esto es de primordial importancia en el sector salud, donde a los recursos limitados se agrega un sistema fragmentado de atención a la población.

Una de las herramientas útiles para dar prioridad a las necesidades y recursos es el indicador de años de vida potencialmente perdidos (AVPP). Este permite evaluar la importancia de una determinada causa de muerte, combinando simultáneamente los criterios de magnitud y temporalidad, ${ }^{3}$ lo que a su vez facilita cuantificar las pérdidas sociales y económicas de una muerte prematura. ${ }^{4}$ Existen diversos métodos para el cálculo de este indicador; algunos autores establecen un intervalo de 0 a 65 años, ${ }^{5}$ otros, de 1 a 70 años, ${ }^{6}$ y otros más consideran desde el nacimiento hasta la esperanza de vida. ${ }^{7}$

Otra metodología, como la desarrollada por $\mathrm{Mu}-$ rray y López, ${ }^{8,9}$ establece un indicador denominado "Años de vida saludable" (AVISA) -Disability adjusted life years, DALY-, producto de un modelo multivariado donde se incluye el tiempo entre la edad que ocurre la muerte y la esperanza de vida, así como una serie de variables relacionadas con la enfermedad y algunas características sociodemográficas de los sujetos que mueren. Sin embargo, no es posible encontrar todos los datos y la tecnología que se requiere para el desarrollo de esta metodología en las microrregiones, lo que dificulta hacer una generalización de los resultados.

En 1994, Bustamante y colaboradore ${ }^{10}$ presentaron los resultados derivados de aplicar el indicador "Años de vida productivos perdidos", según el modelo de inversión-producción-consumo (AVPPipc). En el estudio se ilustra, para el caso de México, el impacto de las diferentes causas de muerte, y se le compara con la importancia relativa de las mismas a partir del ordenamiento entre tasas de mortalidad y el indicador AVPPipc. Sin embargo, sólo se presentan datos en for- ma global para el país, sin tomar en cuenta las diferencias regionales e intrarregionales existentes; considerarlas ayudaría a fijar los objetivos para una toma de decisiones más adecuada y para que los directivos de los servicios de salud movilicen los recursos disponibles.

En una primera aproximación a esta problemática, el presente trabajo expone los resultados del ordenamiento de la mortalidad para cada una de las entidades federativas de la región noroeste de México, utilizando el indicador AVPPipc.

\section{Material y métodos}

Se utilizó la base de datos sobre mortalidad elaborada por el Instituto Nacional de Estadística, Geografía e Informática (INEGI) para el año de 1995. Se seleccionaron las cuatro entidades federativas que conforman el noroeste de México, de acuerdo con la regionalización del Instituto Mexicano del Seguro Social (Baja California, Baja California Sur, Sonora y Sinaloa). Los fallecimientos fueron analizados a partir de las principales causas de mortalidad en la región, según la agrupación que hace la Secretaría de Salud para 1995, con base en la IX Clasificación Internacional de Enfermedades:

- Enfermedades del corazón (E25-E28)

- Tumores malignos (E08-E14)

- Accidentes (E47-E53)

- Diabetes mellitus(E181)

- Enfermedad cerebrovascular (E29)

- Ciertas afecciones originadas en el periodo perinatal (E45)

- Neumonía e influenza (E321-E322)

- Cirrosis y otras enfermedades crónicas del hígado (E347)

- Homicidio y lesiones infligidas intencionalmente por otra persona (E55)

- Nefritis, síndrome nefrótico y nefrosis (E350)

- Enfermedades infecciosas intestinales (E01)

- Anomalías congénitas (E44)

Se siguieron los pasos descritos por Bustamante y colaboradores ${ }^{10}$ para ponderar las defunciones según un balance entre la inversión, la producción y el consumo potencial de cada individuo. En primer lugar se estimó el peso de la enfermedad o pérdida potencial (PP), utilizando como base la esperanza de vida de cada uno de los grupos de edad y la etapa de productividad de los individuos que fallecieron; enseguida se calcularon los años de vida potencialmente perdidos (AVPP) de cada grupo de edad, tomando como 
referencia la esperanza de vida. Por último, se ponderaron los AVPP multiplicándolos por la PP, para obtener los AVPPipc .

\section{Resultados}

En el cuadro I se presentan, para cada una de las entidades federativas y por grupos de edad en que ocurre la muerte, los valores de la esperanza de vida y el resultado del cálculo de la PP. Se puede observar que, en las cuatro entidades federativas, el grupo de edad con mayor pérdida potencial es el de 5 a 14 años y que ésta resulta mayor en los estados de Baja California y Sonora.

En el cuadro II se muestran los resultados del cálculo de los AVPPipc para las cuatro entidades federativas de la región noroeste. Cabe resaltar que en tres de estas entidades, la causa "Ciertas afecciones originadas en el periodo perinatal" (E45) se encuentra ocupando el primer lugar con el indicador AVPPipc; asimismo, tomando en cuenta sólo la tasa cruda, estaría en el sexto lugar de las primeras causas de muerte. Por otro lado, en Sinaloa los homicidios y lesiones infligidas intencionalmente por otra persona (E55) ocupan el primer lugar con el indicador AVPPipc, mientras que considerando las tasas crudas alcanzan el quinto lugar.

Las enfermedades infecciosas intestinales (E01) se ubican, según las tasas crudas, después del décimo lugar en las entidades estudiadas; sin embargo, de acuer- do con los AVPPipc, esta causa se desplaza quinto lugar en Baja California, Baja California Sur y Sonora.

\section{Discusión}

El estudio de la mortalidad nos permite un acercamiento a fenómenos de diversa índole:

1. Demográficos, en tanto se pueden observar las variaciones por edad, sexo, región geográfica y a lo largo del tiempo. ${ }^{11}$

2. Socioeconómicos, dado que es posible relacionar las características socioculturales de la población y la intervención de ésta en la producción y el consumo, así como las relaciones comunitarias y familiares. $^{12}$

3. Epidemiológicos, ya que se puede asociar la mortalidad poblacional e individual con la exposición a factores de riesgo, así como con la patología derivada y concomitante; con causas directas. ${ }^{13}$

Este trabajo retoma la metodología de una investigación de alcance nacional y pretende hacer una aproximación al estudio de la mortalidad regional -en este caso del noroeste de México-, con la utilización del indicador AVPPipc. La importancia de regionalizar con base en este indicador está en que permite, por un lado, reconocer diferencias entre diversas zonas del país y, por el otro, reordenar estas diferencias, las cuales permanecían encubiertas por el orden esta-

Cuadro I

EsPeranza de VIDA Y PÉRDIDA POTENCIAL, SEGÚN GRUPO DE EDAD EN EL QUE OCURRE LA MUERTE. Noroeste de MÉXICO, 1995

\begin{tabular}{|c|c|c|c|c|c|c|c|c|}
\hline \multirow[b]{2}{*}{ Grupos de edad } & \multicolumn{2}{|c|}{ Baja California } & \multicolumn{2}{|c|}{ Baja California Sur } & \multicolumn{2}{|c|}{ Sinaloa } & \multicolumn{2}{|c|}{ Sonora } \\
\hline & $\begin{array}{l}\text { Esperanza } \\
\text { de vida }\end{array}$ & $\begin{array}{l}\text { Pérdida } \\
\text { potencial }\end{array}$ & $\begin{array}{l}\text { Esperanza } \\
\text { de vida }\end{array}$ & $\begin{array}{l}\text { Pérdida } \\
\text { potencial }\end{array}$ & $\begin{array}{l}\text { Esperanza } \\
\text { de vida }\end{array}$ & $\begin{array}{l}\text { Pérdida } \\
\text { potencial }\end{array}$ & $\begin{array}{l}\text { Esperanza } \\
\text { de vida }\end{array}$ & $\begin{array}{c}\text { Pérdida } \\
\text { potencial }\end{array}$ \\
\hline$<1$ & 72 & 28.5 & 76 & 24.5 & 76 & 24.5 & 73 & 27.5 \\
\hline 1 a 4 & 73 & 30 & 75 & 28 & 75 & 28 & 73 & 30 \\
\hline 5 a 14 & 69 & 41 & 71 & 39 & 71 & 39 & 69 & 41 \\
\hline 15 a 24 & 59 & 41 & 62 & 38 & 61 & 39 & 69 & 31 \\
\hline 25 a 34 & 60 & 10 & 62 & 8 & 62 & 8 & 60 & 10 \\
\hline 35 a 44 & 41 & -1 & 43 & -3 & 43 & -3 & 41 & -1 \\
\hline 45 a 54 & 32 & -22 & 34 & -24 & 34 & -24 & 32 & -22 \\
\hline 55 a 64 & 24 & -44 & 25 & -45 & 26 & -46 & 23 & -43 \\
\hline 65 a 74 & 16 & -46 & 17 & -47 & 17 & -47 & 16 & -46 \\
\hline 75 a 84 & 10 & -30 & 12 & -32 & 11 & -31 & 10 & -30 \\
\hline $85 y+$ & 8 & -20 & 7 & -19 & 7 & -19 & 6 & -18 \\
\hline
\end{tabular}

Fuente: cálculos con base en indicadores de mortalidad elaborados por el Instituto N acional de Estadística, Geografía e Informática para 1995 


\section{Cuadro II

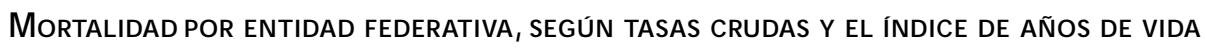

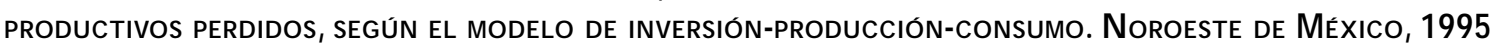

\begin{tabular}{|c|c|c|c|c|c|c|c|c|c|}
\hline \multirow[t]{2}{*}{ Causas de mortalidad } & \multirow{2}{*}{$\begin{array}{l}\text { Clave CIE } \\
\text { (9a. rev.) }\end{array}$} & \multicolumn{4}{|c|}{ Tasa cruda* } & \multicolumn{4}{|c|}{ AVPPipc } \\
\hline & & $\begin{array}{c}\text { Baja } \\
\text { California }\end{array}$ & $\begin{array}{l}\text { Baja California } \\
\text { Sur }\end{array}$ & Sinaloa & Sonora & $\begin{array}{c}\text { Baja } \\
\text { California }\end{array}$ & $\begin{array}{l}\text { Baja California } \\
\text { Sur }\end{array}$ & Sinaloa & Sonora \\
\hline Enfermedades del corazón & E25-E28 & 75.0 & 74.4 & 70.7 & 106.9 & -51027 & -8359 & -61456 & -68543 \\
\hline Tumores malignos & E08-E14 & 56.0 & 52.1 & 64.0 & 69.7 & -33487 & -6839 & -44480 & -41282 \\
\hline Accidentes & E47-E53 & 48.1 & 42.2 & 45.7 & 48.9 & $9897^{\ddagger 2}$ & $1296^{\ddagger 2}$ & $4328^{\ddagger 3}$ & $3201^{\ddagger 3}$ \\
\hline Diabetes mellitus & E81 & 37.0 & 32.5 & 27.7 & 38.4 & -27527 & -3965 & -23999 & -29071 \\
\hline Enfermedad cerebrovascular & E29 & 24.9 & 16.1 & 23.3 & 26.3 & -14894 & -2071 & -16964 & -14963 \\
\hline $\begin{array}{l}\text { Ciertas afecciones originadas en } \\
\text { el periodo perinatal }\end{array}$ & E45 & 25.6 & 18.3 & 7.8 & 20.3 & $14364^{\ddagger 1}$ & $1984^{\ddagger 1}$ & $4434^{\ddagger 2}$ & $10368^{\ddagger 1}$ \\
\hline N eumonía e influenza & E321,E322 & 16.8 & 15.3 & 9.5 & 13.9 & -626 & -1017 & -3880 & -3231 \\
\hline $\begin{array}{l}\text { Cirrosis y otras enfermedades } \\
\text { crónicas del hígado }\end{array}$ & E347 & 19.6 & 12.1 & 7.8 & 13.0 & -8849 & -1258 & -6171 & -7350 \\
\hline $\begin{array}{l}\text { Homicidio y lesiones infligidas } \\
\text { intencionalmente por otra persona }\end{array}$ & E55 & 15.9 & 7.0 & 25.9 & 12.5 & $5116^{\ddagger 4}$ & $340^{\ddagger 4}$ & $5296^{\ddagger 1}$ & $2334^{\ddagger 4}$ \\
\hline N efritis, síndrome nefrótico y nefrosis & E350 & 8.9 & 7.0 & 7.9 & 9.8 & -5022 & -902 & -5892 & -5662 \\
\hline Enfermedades infecciosas intestinales & E01 & 5.3 & 2.7 & 4.2 & 9.2 & $1700^{\ddagger 5}$ & $77^{\ddagger 5}$ & -1126 & $392^{\ddagger 5}$ \\
\hline A nomalías congénitas & E44 & 10.8 & 12.1 & 5.8 & 8.6 & $6262^{\ddagger 3}$ & $611^{\ddagger 3}$ & $3205^{\ddagger 4}$ & $4469 \neq 2$ \\
\hline
\end{tabular}

Fuente: Indicadores de mortalidad elaborados por el Instituto N acional de Estadística, Geografía e Informática para 1995 y cálculos hechos con base en ellos

blecido a partir de las tasas crudas de mortalidad. Así, una región noroeste aparentemente homogénea en la mortalidad por problemas crónico-degenerativos, tumorales y accidentes, muestra discrepancias entre las entidades federativas que la conforman. Sin embargo, es preciso señalar que en las regionalizaciones hay que tener cuidado con los límites y divisiones, ya que, como señalan Kunz y colaboradores, ${ }^{14}$ los límites deben lograr una generalidad máxima con el menor número de divisiones posible, pero sin perder demasiado detalle.

En este estudio resalta el hecho de que, al ponderar la mortalidad considerando un valor económico a la edad en que ocurrió el fallecimiento (AVPPipc), se reordenan los cinco primeros lugares que ahora son ocupados por: a) las afecciones originadas en el periodo perinatal, b) los accidentes, c) las anomalías congénitas, d) los homicidios y e) las enfermedades infecciosas intestinales.

Así, este indicador modifica el patrón epidemiológico ydemográfico que, a partir de tasas crudas de mortalidad, colocaba a las enfermedades crónico-de- generativas y tumorales entre los primeras causas en estas entidades federativas. ¿Qué implica esto? En primera instancia, que quienes toman decisiones relacionadas con los servicios y políticas de salud, así como los encargados de las áreas de difusión y promoción de la salud, pueden considerar la utilización óptima de recursos en función de esta nueva dimensión de la mortalidad.

En segundo término, esta metodología permite observar que algunos problemas como las muertes violentas o los homicidios, por ejemplo, tienen un peso mayor en la población económicamente activa, y que esto ocasiona múltiples consecuencias personales, familiares, institucionales y sociales. Ello conduce a poner énfasis en la prevención y la atención de ciertas patologías poblacionales. Es decir, si con el indicador AVPPipc los problemas crónico-degenerativos y tumorales descienden de lugar, como causa primera de mortalidad, esto se debe a que así se pondera la importancia de los años perdidos con el peso económico que ello representa para la sociedad, tanto si el fallecimiento ocurre en el momento de la "inversión social", 
es decir, en la infancia y el primer año de vida, como si sucede en la etapa económicamente activa, es decir en el momento de la "producción social".

Un tercer elemento de importancia, tanto para los encargados de tomar decisiones como para los investigadores en sistemas de salud, tiene que ver con el análisis de los procesos de prevención de factores de riesgo y calidad de la atención de los servicios de salud. En efecto, el reordenamiento de las causas de mortalidad generado por la ponderación con el AVPPipc, ofrece la visión de una mortalidad por afecciones congénitas y por aquellas originadas en el periodo perinatal, lo que incide sobre la cobertura y la calidad de los programas de salud materno-infantil. Dicho de otra forma, este reordenamiento de la mortalidad puede dar a los encargados de tomar decisiones, directivos y administradores, así como al personal de salud operativo de las instituciones, una dimensión diferente en el análisis de los programas y políticas de salud reproductiva.

En cuanto al análisis de la mortalidad con la utilización del AVPPipc, se observó que las causas se reordenan, con lugares positivos y negativos, de forma similar en las cuatro entidades federativas. Esto es, los problemas crónico-degenerativos y tumorales de la población adulta mayor adoptan un valor negativo, y las causas perinatales, así como las violentas, adoptan un valor positivo. Esto sugiere que, muy probablemente, las condiciones socioeconómicas y de calidad de los servicios de salud en las cuatro entidades federativas son similares.

Sin embargo, Sinaloa mostró un comportamiento diferente en relación con las otras entidades que conforman la región noroeste, pues la mortalidad por homicidios tuvo el mayor valor positivo, y las enfermedades infecciosas intestinales, un valor negativo. Estas observaciones surgen ahora como nuevas interrogantes de estudio para análisis posteriores, y lo interesante es que surgen a partir de haber hecho ponderaciones con los AVPPipc. Este indicador puede ser utilizado fácilmente en los servicios e instituciones de salud y seguridad social. El algoritmo de cálculo es sencillo de contabilizar con una computadora personal y una hoja de cálculo accesible a cualquier administrador de hospital o de servicios de salud.

Los resultados en este estudio coinciden con los alcanzados por Lozano y colaboradores ${ }_{1}^{15}$ quienes encontraron que la mortalidad por homicidios en el estado de Sinaloa tiene un diferencial entre la población económicamente activa, respecto a las otras tres entidades. No obstante, cabe aclarar que la metodología utilizada en el presente trabajo es más sencilla.

Por último, los autores desean resaltar el hecho de que adoptar un indicador de años de vida poten- cialmente perdidos con una ponderación económica no conduce, per se, a tomar mejores decisiones o a hacerlo con un sentido humanista. Este indicador es una herramienta de trabajo, al igual que la tecnología educacional en salud o las estrategias de calidad de la atención, la planeación estratégica o, incluso, algún nuevo medicamento o vacuna, todos ellos productos del saber humano que pueden -y deberían- incidir en el mejoramiento de la salud en las poblaciones.

\section{Referencias}

1. Jiménez-0 rnelas RA. La desigualdad de la mortalidad para la República Mexicana y sus entidades federativas 1990. C uernavaca, Mor.: Centro Regional de Investigaciones Multidisciplinarias-Universidad $\mathrm{N}$ acional Autónoma de México, 1995: 9-11.

2. López-Cervantes M. Utilización de los datos de mortalidad para la planeación de los servicios de salud: el caso de México. Salud Publica Mex 1985;27:124-139.

3. Izazola-Licea JA,Valdez-G arcía M, Sánchez-Pérez HJ, D el Río-Chiriboga C. La mortalidad por el SIDA en México de 1983 a 1992. Tendencias y años perdidos de vida potencial. Salud Publica Mex 1995;37:140-148.

4. Gardner JW, Sanborn JS.Years of potential life lost (YPLL).W hat does it measure? Epidemiology 1990;1(4):322-329.

5. MMW R. Years of potential life lost before age 65.U nited States, 1990 and 1991. MMW R Morb Mortal W kly Rep 1993;42(13):251-253.

6. Romeder JM, Mc W hinnie JR. Potential years of life lost between ages 1 and 70:An indicator of premature mortality for health planning. Int J Epidemiol 1977;6(2):143-151.

7. Mingot $M$, Rue M, Borrell C.Years of potential life lost: C omparison of 3 calculation methods. Gac Sanit 1991;5(22):21-28.

8. Murray CJL, López AD. G lo bal mortality, disability, and the contribution of risk factors: Global burden of disease study. Lancet 1997;349:1436-1442.

9. Murray CJL, Lopez AD. A Iternative projections of mortality and disability by cause 1990-2020: Global Burden of Disease Study. Lancet 1997; 349:1498-1504

10. Bustamante-Montes LP, Rascón-Pacheco RA, Borja-Aburto VH. Efectos de la aplicación del indicador de años de vida productivos perdidos (modelo de inversión producción consumo) en el ordenamiento de las causas de muerte en México 1990. Rev Saude Publica 1994;28(3): 198-203.

11. Sepúlveda J, coord. La mortalidad en México; registro, estructura y tendencias.México, D.F.: Secretaría de Salud (Cuadernos de Salud, Serie Información en Salud, núm. 1), 1994.

12. G arcía-Molina C, Hernández-Bringas $\mathrm{H}$, coord. Mortalidad, salud y discurso demográfico. Cuernavaca, Mor.: C entro Regional de Investigaciones Multidisciplinarias-Universidad Nacional Autónoma de México, 1996.

13. Puffer, Ruti R. N ew approches for epidemiology studies of mortality statistics. Bull Pan Am Health 0 rgan 1989;(23)4:365-383.

14. KunzY, Cortina M, González-Block MA. Regionalización socioeconómico-demográfica y de salud de la República Mexicana: un instrumento para la planeación e investigación de atención primaria a la salud. México, D.F.: Instituto $N$ acional de Salud Pública (Perspectivas en Salud Pública, núm. 2), 1986.

15. Lozano R, Híjar M,Torres JL.Violencia, seguridad pública y salud. En: Frenk J, ed. 0 bservatorio de la salud. Necesidades servicios políticas. México, D.F.: Fundación Mexicana para la Salud, 1997:83-115. 